\title{
Potencial económico de la captura de peces de ornato bajo protección federal en México
}

\author{
Diego Gijón-Díaz ${ }^{1}$, Héctor Reyes-Bonilla ${ }^{1}$, Triana P. Guerrero-Izquierdo ${ }^{1} \&$ \\ Francisco J. Fernández-Rivera Melo ${ }^{2}$ \\ 1. Universidad Autónoma de Baja California Sur. Carretera al Sur km 5.5. Col. El Mezquitito. C. P. 23080. La Paz, B. \\ C. S; dgijon26@gmail.com, hreyes@uabcs.mx, trianapaulinagi@hotmail.com \\ 2. Comunidad y Biodiversidad, A.C. Calle Isla del Peruano \# 215 Col. Lomas de Miramar. C. P. 85448. Guaymas \\ Sonora, México; ffernandez@cobi.org.mx
}

\section{Recibido 28-I-2016. Corregido 03-VIII-2016. Aceptado 02-IX-2016.}

\begin{abstract}
Economic potential of ornamental fish catching under federal protection in Mexico. Ornamental fisheries are economically viable activities in developing countries due to the fact that aquaria have become a worldwide hobby. In Mexico, the fishery has been conducted since the 1980s, especially in the Baja California Peninsula, but nevertheless, there are no adequate records of commercial production, or about the economic gain due to the exploitation of the resource. The objective of this paper was to calculate the maximum allowable catch for the five ornamental fishes included in the NOM-059-SEMARNAT (protected by federal mandate), and the net monetary gain that the resource might generate. Censuses $(\mathrm{N}=1610)$ were conducted in 21 reef sites of the Mexican Pacific and Gulf of California in order to estimate population density of each taxon; also, natural mortality rate $(\mathrm{M})$ was calculated from maximum size $(\mathrm{L} \infty)$, individual growth rate $(\mathrm{k})$ and temperature (C), and the total allowable catch was estimated on the basis of M. Finally, considering the prices of the species paid to fishermen at the beach, an estimation of the economic value of a single fishing season for an area of 220 hectares (representative of the catch area assigned in average to a cooperative in Mexico) was produced. The results indicated that the areas with the best economic potential were Encantadas Islands, Los Angeles Bay, and San Lorenzo Archipelago (all in the state of Baja California). Outside of the gulf (where no legal ornamental fisheries exist) the potentially more rentable locations were Marietas Islands (Jalisco), and the Revillagigedo Islands. Aquarium fisheries may be a viable activity in several of the analyzed areas of the country, however, the potential gain that harvest represents is not enough to fully sustain an organized group that would perform it; for that reason the ornamental fishery should be seen as an alternative commercial activity. Finally, to guarantee this activity to be a rentable and sustainable fishery, we recommended that, the existing rules defined by the Mexican federal government should be applied to control the catch quotas. Rev. Biol. Trop. 65 (1): 195-210. Epub 2017 March 01.
\end{abstract}

Key words: Holacanthus clarionensis, Holacantus passer, Pomacanthus zonipectus, Chromis limbaughi, Opistognathus rosenblatti, MAQTRAC.

Las especies marinas de importancia ornamental son aquellas que se capturan para mantenerse vivas en acuarios o estanques; su valor y su uso están dirigidos a la belleza escénica que sus formas y vivos colores producen (PiñaEspallargas et al., 2000). La industria del acuarismo marino inició en Filipinas en los años 1950s y desde entonces se han hecho esfuerzos a nivel mundial para generar conocimientos necesarios para el cuidado y mantenimiento de peces en cautiverio, y de asegurar la forma correcta para la exportación de peces de acuario (Albaladejo \& Corpuz, 1981).

A mediados de los 1970s se registraron en México las primeras capturas formales de especies marinas para su comercialización en la industria de la acuariofilia, pero la extracción no era considerada de relevancia (Piña-Espallargas et al., 2000). En 1995, la Ley de Pesca y su Reglamento instauraron el marco legal 
para el aprovechamiento de este recurso basado en el Permiso de Pesca de Fomento otorgado por la Secretaría de Agricultura, Ganadería, Desarrollo Rural, Pesca y Alimentación (SAGARPA) a través de la Comisión Nacional de Acuacultura y Pesca (CONAPESCA), y se determinó que las capturas podrían tener dos destinos: acuarios educativos y el mercado del acuarismo. A la fecha, en México la mayoría de las especies de ornato son capturadas bajo el citado esquema de pesca de fomento, a excepción de cinco de ellas (Secretaría de Medio Ambiente y Recursos Naturales, 2012): Pomacanthus zonipectus (Gill, 1862) (Ángel de Cortés), Holacanthus passer (Valenciennes, 1846) (Ángel rey), Holacanthus clarionensis (Gilbert, 1890) (Ángel Clarión), Chromis limbaughi (Greenfield y Woods, 1980) (Damisela azul-amarillo) y Opistognathus rosenblatti (Allen y Robertson, 1994) (Bocón puntos azules). Estas se caracterizan por tener el mejor precio de mercado y representan la mayor parte de las capturas registradas (Piña-Espallargas et al., 2000, Reyes-Bonilla, Herrero-Pérezrul, \& Fernández-Rivera, 2009); por esa razón se extraen dentro de Predios Federales de Aprovechamiento, siguiendo directivas definidas por la Ley General de Vida Silvestre (LGVS), ya que todos se encuentran incluidos en la NOM-059-SEMARNAT- 2010, en la categoría de "Protección especial". Esta última abarca aquellas especies que podrían llegar a encontrarse amenazadas por factores que inciden negativamente en su viabilidad, por lo que se determina la necesidad de propiciar su recuperación y conservación (SEMARNAT, 2012).

Los 1990s ofrecieron grandes expectativas para el acuarismo marino, debido a la existencia de mayor número de aficionados, tecnología más avanzada, accesorios modernos, sal preparada para acondicionar los acuarios domésticos y suplementos alimenticios balanceados (Piña-Espallargas, 2005). A partir de esa década la pesca de ornato se convirtió en una industria multimillonaria que ha incrementado a una tasa anual superior al $10 \%$ a partir de 1985 (Food and Agriculture Organization of the United Nations, 1999; Ramirez-Martínez et al., 2010). Es difícil estimar estadísticas globales sobre el comercio de peces de ornato, pero se menciona que esta industria involucra a más de 100 países y que puede generar hasta 20 mil millones de dólares americanos (RamirezMartínez et al., 2010). Han, et al. (2002), realizaron un estudio sobre aspectos económicos de la industria de peces ornamentales en Hong Kong, y describieron su evolución; en el trabajo se condujo una revisión de los establecimientos de venta de estos organismos de 1978 hasta 2001 y se documentó la zona de Goldfish Street como aquella que tiene la concentración más importante de tiendas. La ganancia neta de esos negocios fue de entre $\$ 7.7$ millones a $\$ 11.5$ millones USD por año.

En México, dado que la extracción de especies marinas ornamentales no ha sido considerada como una pesquería, no existe información de la producción nacional en los Anuarios Estadísticos de Pesca; por ello no se conoce el volumen real de capturas ni el lugar que ocupa México a nivel mundial en este rubro. Lo que se sabe es que en el país, cerca del $98 \%$ del producto de ornato se exporta a los Estados Unidos, y el resto se comercializa a nivel nacional, principalmente en las ciudades de México y Guadalajara (Piña-Espallargas, 2005). La extracción de especies de acuario se caracteriza por ser multiespecífica (ReyesBonilla et al., 2009), y es interesante denotar que el volumen de las capturas no depende directamente de la abundancia estacional de las especies, o de los envíos por parte de los productores (es decir, de la oferta), sino de las solicitudes que se hagan de los organismos por parte de los intermediarios, es decir, de la demanda (Piña-Espallargas, 2000). En el Golfo de California se capturan aproximadamente 320 especies (aproximadamente el $5 \%$ de las especies marinas que se extraen en el mundo), un total de 150 corresponden a peces y 170 a invertebrados (Piña-Espallargas, 2000), y como se dijo, la pesquería se concentra en las cinco especies presentes en la Norma Oficial Mexicana 059-SEMARNAT, 2010. Cabe señalar que esta región es la única en México donde se cuenta con permisos oficiales de captura 
de especies de ornato, todos a lo largo de la Península de Baja California (M. A. CastilloCeja, Titular de la Unidad de Enlace SEMARNAT, Comunicación personal, Julio de 2010).

En 2012 la SEMARNAT presentó un Plan de Manejo Tipo para Especies Marinas Ornamentales, el cual está enfocado en la conservación y aprovechamiento sustentable de los peces de acuario, y describe los elementos necesarios para llevar a cabo buenas prácticas, aprovechar sustentablemente el recurso y fomentar su conservación, para mantener en mente la necesidad de emplear la pesca de ornato como un mecanismo para impulsar beneficios socio-económicos que incluye la generación de empleos en las comunidades pesqueras, y fortalecer el precio de venta del producto, dada la buena calidad del mismo (SEMARNAT, 2012). Estas ordenanzas surgen del hecho que aunque en México ya existe la comercialización formal, las regulaciones anteriores eran muy generales y además se habla de una intensa captura ilegal (Almenara-Roldán \& Ketchum, 1994), situación que abarata el precio en el mercado regulado, y obliga a que diversas agencias además de SAGARPA y DGVS ayuden a la regulación de la actividad (Lango-Reynoso et al., 2012), que incluye a la Comisión Nacional de Áreas Naturales Protegidas (CONANP), la Secretaría de Marina (SEMAR), y la Procuraduría Federal de Protección al Ambiente (PROFEPA).

Para México hay muy pocos trabajos enfocados al análisis de la pesquería de ornato. A partir de observaciones en años repetidos de visita, Almenara-Roldán \& Ketchum (1994) mencionan que la pesca ilegal en el Archipiélago de Revillagigedo debe ser importante, y como resultado estimaron una disminución en el tamaño de las poblaciones del pez Ángel Clarión en dicha región. Por otra parte, Almenara-Roldán (2000) realizó un estudio acerca de la demanda internacional que tienen las especies ornamentales del Golfo de California y encontró que entre las más importantes dentro de esta actividad destacan las endémicas: el Bocón gigante (Opistognathus rhomaleus Jordan y Gilbert, 1881), el Bocón puntos finos
(Opistognathus punctatus Peters, 1869), y el Bocón puntos azules (Opistoganthus rosenblatti). También se mencionó a Pomacanthus zonipectus el Ángel de Cortés y Holocanthus passer (Ángel Rey) como dos de las especies más solicitadas por los acuaristas del mundo. Finalmente, el reporte menciona como un caso especial a Holocanthus clarionensis, ya que es de suma importancia comercial pero no se trata de una especie común del Golfo de California sino que es habitante de las Islas Revillagigedo, y ocasionalmente es encontrado entre La Paz y Los Cabos.

Sala \& Sánchez-Ortiz (2000) sugieren la realización de investigaciones científicas aplicadas a la gestión de las especies marinas de ornato en el Golfo de California, y proponen enfocarse a la conducción de estudios sobre la reproducción en cautiverio de las especies marinas de ornato, que ayuden a la conservación de poblaciones de peces de arrecife. Más recientemente, Reyes-Bonilla et al. (2009) realizaron un estudio sobre aspectos económicos de los recursos pesqueros no tradicionales en zonas de arrecifes en el Golfo de California donde describen el modo de operación, las características de comercialización, y presentan algunas estadísticas del mercado de las especies de ornato. Los autores concluyen que hay muy poca información como para estimar el efecto que la pesca ha tenido sobre las poblaciones, pero que este debe ser bajo, dado que rara vez se cubren en su totalidad las cuotas asignadas a los Predios Federales de Aprovechamiento que trabajan este recurso en Baja California. Finalmente, Germain, Hartmann, FernándezRivera \& Reyes-Bonilla (2015) demostraron que la venta de peces de ornato por parte de la cooperativa "Mujeres del Golfo", ha dado a las socias un avance económico importante, así como empoderamiento social, al pasar a ser elementos reconocidos en su localidad e incluso fuera de ella, gracias a algunos programas de televisión que han descrito sus operaciones.

Como se ha mencionado, la pesca de especies marinas de ornato es una industria de millones de dólares, que soporta a miles de pescadores en los países en desarrollo 
(Wood, 2001). En México se busca la forma de ayudar al desarrollo ordenado de la actividad, de modo que pueda haber captura pero sin alterar notablemente a las poblaciones (ReyesBonilla et al., 2009). Para lograr esto se requiere tener información no solo de las pesquerías actuales, sino también sobre la abundancia del recurso en diversas zonas del país, y del potencial económico que puedan arrojar ahí donde exista la posibilidad para el desarrollo adecuado de la pesquería. Por lo anterior, en el presente trabajo se generaron datos de densidad poblacional de las cinco principales especies de ornato de México (todas residentes exclusivas de la costa occidental del país, y bajo protección federal al estar presentes en la NOM-059-SEMARNAT 2010), se estimó un nivel aceptable de captura en diversas zonas arrecifales a lo largo de la región, y se calculó el potencial económico que su captura podría arrojar para las comunidades locales.

\section{MATERIALES Y MÉTODOS}

Trabajo de campo: Para cuantificar la abundancia de las cinco especies de peces de interés (Chromis limbaughi, Holacanthus passer, H. clarionensis, Opistognathus rosenblatti y Pomacanthus zonipectus), se realizaron 1610 censos visuales de banda mediante la metodología del Plan de Manejo Tipo para Peces de Ornato en México (SEMARNAT, 2012), también descrita por Chávez-Comparán et al. (2010). Los conteos se efectuaron en 21 localidades arrecifales del Pacifico Mexicano y Golfo de California (Fig. 1) entre Julio 2009 y Octubre 2010, y se condujeron dentro de las zonas arrecifales rocosas y coralinas, y a profundidades de 3 a $15 \mathrm{~m}$, que es la zona donde las especies de interés normalmente se encuentran (Thomson, Findley, \& Kerstitch, 2000). Los recorridos en el Pacífico se realizaron dentro de bandas de observación de 30 de largo x $2 \mathrm{~m}$ de ancho, mientras que en el Golfo de California las unidades de muestreo ocuparon $25 \times 4 \mathrm{~m}$. De la información obtenida, para cada especie se calculó la densidad por transecto (se dividieron los organismos observados entre el área del censo, arrojando unidades de indiv. $/ \mathrm{m}^{2}$ ), luego el promedio general por localidad (tomando en cuenta todos los conteos realizados en cada sitio), y a partir de ahí se calculó la abundancia total por hectárea, al emplear extrapolación directa (es decir, se multiplicó la densidad en indiv. $/ \mathrm{m}^{2}$ por el número de metros en una hectárea del hábitat arrecifal). Todos los censos se realizaron en las temporadas de primavera y otoño para aprovechar la transparencia de las aguas y hacer más eficiente la labor de censo, y además cabe señalar que las cinco especies bajo análisis son relativamente longevas, y por ello sus abundancias no varían mucho a lo largo del año (Pérez-España, Galván-Magaña, \& Abitia-Cárdenas, 1996).

Para todas las especies se realizaron análisis estadísticos con el fin de determinar si había diferencias significativas en la densidad por localidad. Como los datos no presentaron normalidad u homoscedasticidad, con este fin se efectuaron pruebas de Kruskal-Wallis (K-W) y sus correspondientes análisis a posteriori de Mann-Whitney (Zar, 2009), con un error alfa de 0.05 .

Análisis pesquero: Con los datos de abundancia obtenidos de los censos, para cada localidad se determinó la cuota potencial admisible para cada especie, siguiendo la metodología MAQTRAC (Hodgson \& Ochavillo, 2006) que se basa en la ecuación: $\mathrm{CMP}=e^{(\mathrm{M})}$, Dónde: $\mathrm{CMP}=$ Máxima captura permisible (en porcentaje de la población presente), $e=$ Constante de Euler, $\mathrm{M}=$ Tasa de mortalidad natural (adimensional).

Para conocer las tasas de mortalidad natural de cada especie, se tomó en cuenta su longitud teórica máxima ("longitud asintótica";

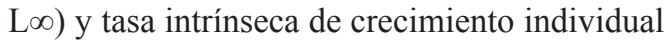
(k), y se aplicó la ecuación empírica de Pauly (1983), empleando el programa FiSAT (Fisheries Assessment Tools), programado por la FAO e ICLARM (Gayanilo, Sparre, \& Pauly, 2005): $\log M=-0.0066-0.279 * \log (\operatorname{L} \infty)+$ $0.6543 * \log (\mathrm{k})+0.4634 * \log (\mathrm{t})$, Dónde: $\mathrm{L} \infty$ $=$ Longitud asintótica $(\mathrm{en} \mathrm{cm}), \mathrm{k}=$ Constante 


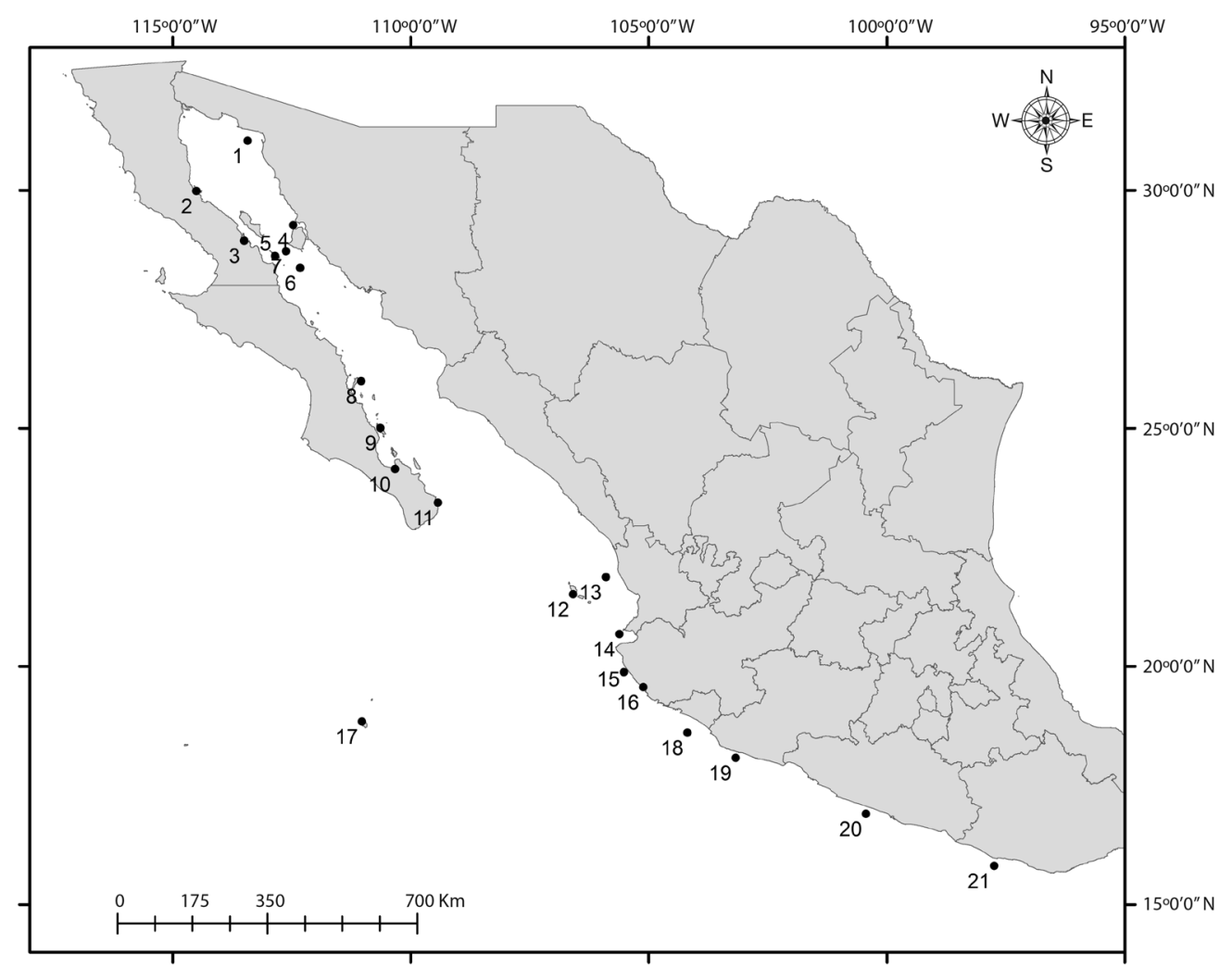

Fig. 1. Área de estudio: 1 (Isla San Jorge), 2 (Islas Encantadas), 3 (Bahía de los Ángeles), 4 (Isla Tiburón), 5 (Archipiélago San Lorenzo), 6 (Isla San Pedro Mártir), 7 (Isla San Esteban), 8 (Loreto), 9 (Isla San José), 10 (Bahía de la Paz), 11 (Cabo Pulmo), 12 (Islas Marías), 13 (Isla Isabel), 14 (Islas Marietas), 15 (Tenacatita), 16 (Chamela), 17 (Manzanillo), 18 (Islas Revillagigedo), 19 (Faro de Bucerías), 20 (Ixtapa), 21 (Huatulco).

Fig. 1. Study area: 1 (Isla San Jorge), 2 (Islas Encantadas), 3 (Bahía de los Ángeles), 4 (Isla Tiburón), 5 (Archipiélago San Lorenzo), 6 (Isla San Pedro Mártir), 7 (Isla San Esteban), 8 (Loreto), 9 (Isla San José), 10 (Bahía de la Paz), 11 (Cabo Pulmo), 12 (Islas Marías), 13 (Isla Isabel), 14 (Islas Marietas), 15 (Tenacatita), 16 (Chamela), 17 (Manzanillo), 18 (Islas Revillagigedo), 19 (Faro de Bucerías), 20 (Ixtapa), 21 (Huatulco).

de crecimiento (adimensional), $\mathrm{t}=$ Temperatura promedio en cada sitio de estudio $\left({ }^{\circ} \mathrm{C}\right)$.

Los parámetros poblacionales de $P$. zonipectus, H. passer y C. limbaughi (Lळ y k) se obtuvieron a partir de mediciones llevadas a cabo en campo en el Parque Nacional Bahía de Loreto $\left(25^{\circ} \mathrm{N}\right)$ en el año 2010, y se emplearon rutinas del programa FiSAT. Para el caso de Opistognathus rosenblatti se tomó directamente el dato de mortalidad natural de la base de datos FISHBASE (Froese \& Pauly, 2014), y para Holacanthus clarionensis, la longitud máxima se calculó mediante la fórmula de Sparre \& Venema (1997): L $\infty=$ longitud máxima medida en el campo / 0.95 .
Los datos de temperatura del océano utilizados para estimar la tasa de mortalidad natural se obtuvieron de la Agencia Aeroespacial Nacional de los Estados Unidos (NASA, por sus siglas en inglés), en la página de internet del Servicio de Visualización en Línea de Datos Radiométricos del Color del Océano (Giovanni, 2013), y fueron generados por el satélite MODIS-Aqua a un tamaño de pixel de $9 \mathrm{~km}$.

Una vez obtenido el porcentaje de la población de cada especie, factible de ser capturada, se calculó el producto de ese valor por la abundancia promedio por hectárea estimada, para cada localidad; esto permitió saber el número de individuos que sería permisible 
pescar en un área de 10000 metros cuadrados de cada sitio. Luego, esta última cifra se multiplicó por su respectivo valor económico (precio en playa) para cada tipo de pez, y así se computó la posible derrama económica bruta que dejaría su captura. El precio de playa para 2013 se obtuvo por cortesía de los responsables técnicos de dos Predios Federales de Aprovechamiento, asignados a las sociedades cooperativas "Mujeres del Golfo" de Ligüi B.C.S., y "Baja Ornaments", de Ensenada B.C. Finalmente, la ganancia bruta se estandarizó a una medida de 220 hectáreas de arrecife; ésta es una extensión pequeña (equivalente a un cuadrado de 1483 metros de lado), sin embargo, se tomó como referencia, ya que es la asignada por la DGVS a la cooperativa "Mujeres del Golfo", la cual ha demostrado gran eficacia en su operación (Germain et al., 2015). A partir de estas cifras se estimó la ganancia neta, que equivale al $21 \%$ del monto, una vez que se han restado los gastos de inversión requeridos para la actividad. Este porcentaje fue proporcionado por las dos cooperativas contactadas. Con la ganancia económica neta que dejaría cada especie de interés si se capturara en cada una de las 21 localidades de análisis, se sumaron los valores de las cinco y se convirtieron a dólares (a la paridad de 13 pesos por dólar) para obtener el valor económico potencial por localidad, que tendría una temporada de pesca, y para cada hectárea de arrecife explotado.

\section{RESULTADOS}

Distribución y densidad poblacional de las especies de interés: Para poder describir nuestros resultados fue primero necesario dar a conocer los sitios donde se realiza la actividad extractiva de especies de ornato (Fig. 2). A partir de los censos en campo se observó que las localidades en donde se puede encontrar la mayoría de las especies de interés fueron Bahía de los Ángeles y Archipiélago San Lorenzo, en Baja California, ya que presentan a Chromis limbaughi, Holacanthus passer, Pomacanthus zonipectus y Opistognathus rosenblatti (Fig. 3).
De los peces analizados, la damisela azulamarillo C. limbaughi tuvo amplia distribución dentro del Golfo de California ya que estuvo presente en ocho sitios, aunque fuera del golfo (en el Pacífico Tropical Mexicano), sólo apareció en Manzanillo, Colima. La especie presentó una densidad promedio de 0.035 indiv./ $\mathrm{m}^{2}$ para la región de estudio completa, siendo Bahía de los Ángeles, B.C. el sitio con el mayor promedio de densidad $(0.151 \pm 0.022$ indiv./ $\left.\mathrm{m}^{2}\right)$, seguido de Islas Encantadas, B. C. (0.129 \pm 0.055 indiv. $\left./ \mathrm{m}^{2}\right)$ y Bahía de La Paz, B. C. S. $\left(0.097 \pm 0.039\right.$ indiv. $\left./ \mathrm{m}^{2}\right)$. El análisis estadístico mostró diferencias significativas entre

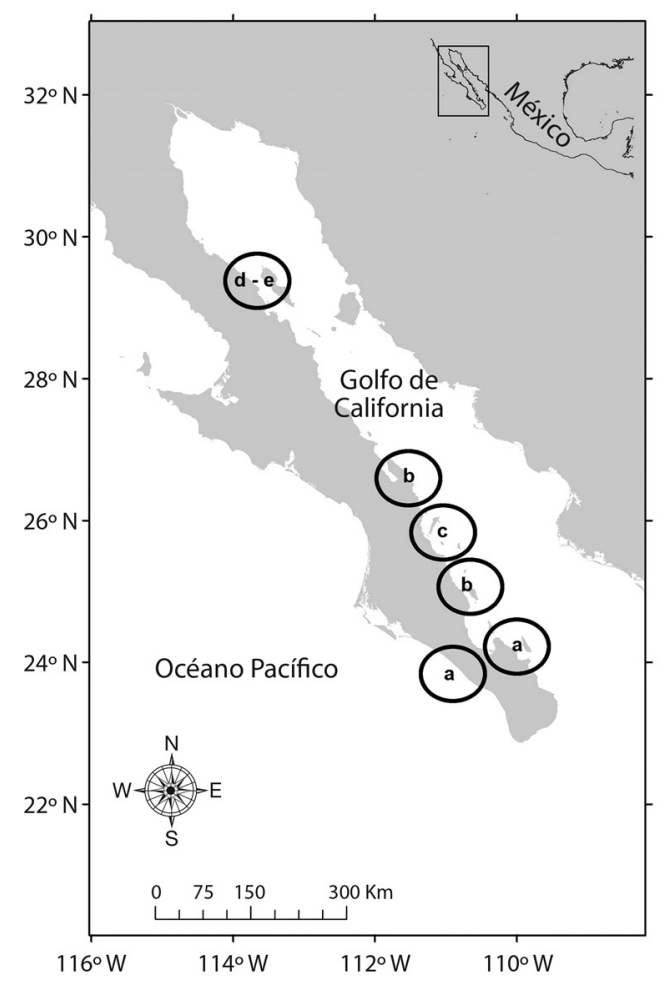

Fig. 2. Zonas con permisos oficiales de captura de especies de peces arrecifales ornato bajo protección federal: a) Buzos del Golfo), b) Arrecife Acuario), c) Cooperativa Mujeres del Golfo.), d) San Rafael y San Francisquito, y e) Mar de Cortés.

Fig. 2. Areas with official permits for the capture of ornamental reef fish species under federal protection. A) Divers Gulf), b) Reef Aquarium), c) Cooperative Women Gulf), d) San Rafael and San Francisquito, e) Sea of Cortez. 

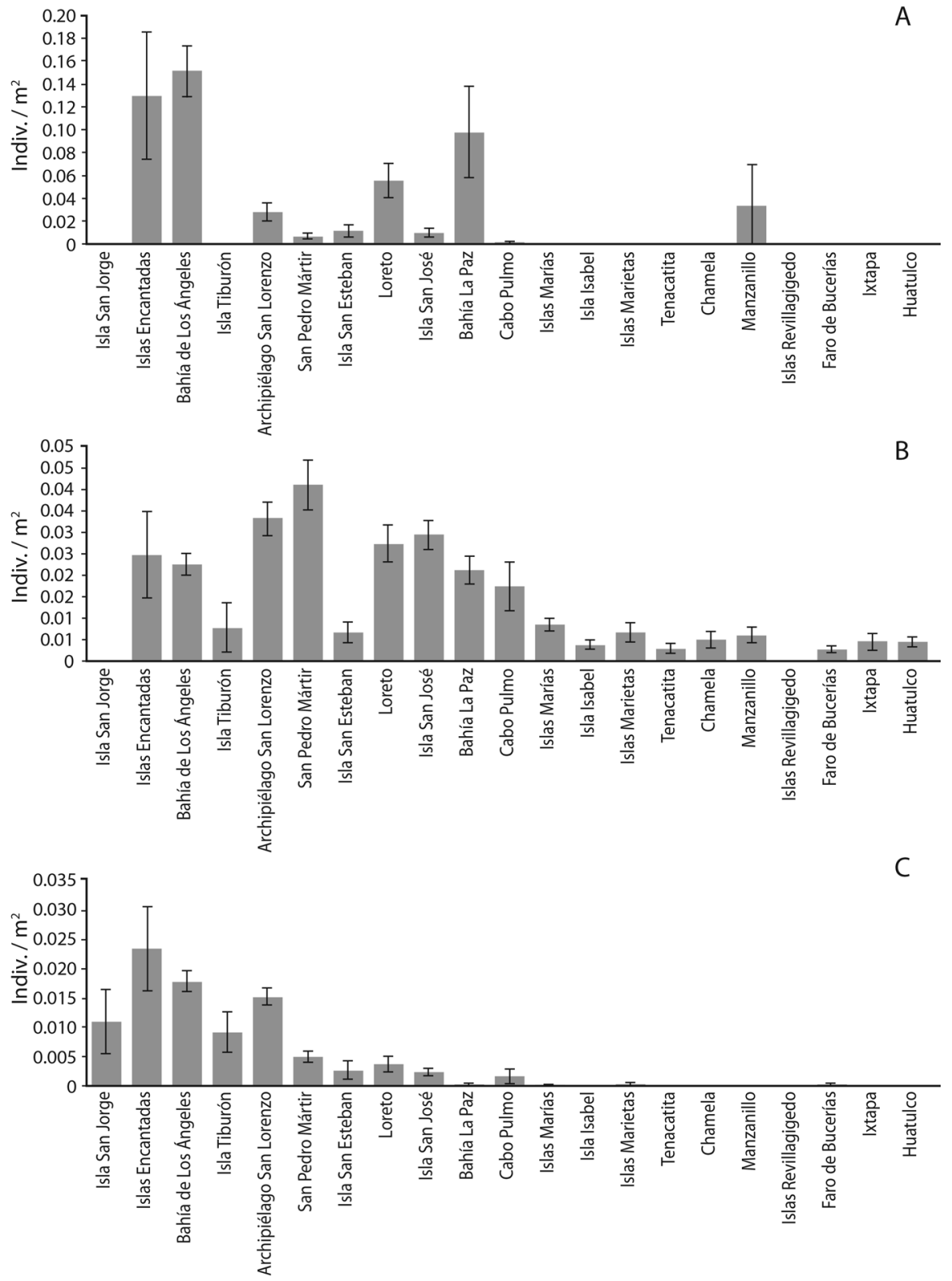

Fig. 3. Densidades poblacionales de: a) Chromis limbaughi, b) Holacanthus passer, y c) Pomacanthus zonipectus, para cada localidad revisada.

Fig. 3. Population densities of: a) Chromis limbaughi, b) Holacanthus passer, and c) Pomacanthus zonipectus, for each surveyed location.

los sitios $\left(\mathrm{K}-\mathrm{W}_{(20,1610)}=267.856, \mathrm{p}<0.001\right.$, Fig. 2) y nos muestra que Bahía de los Ángeles, Islas Encantadas y La Paz, los sitios que resultaron diferentes del resto, de acuerdo a la prueba a posteriori.
El ángel rey, H. passer, presentó la más amplia distribución geográfica de los taxa bajo estudio en el Pacífico Mexicano (promedio de densidad de $0.018 \pm 0.001$ indiv. $/ \mathrm{m}^{2}$ a lo largo de toda la región (Fig. 3), aunque tuvo mayor 
ocurrencia hacia el norte (dentro del Golfo de California). Los sitios con mayor densidad de ésta especie fueron San Pedro Mártir, Sonora $\left(0.040 \pm 0.005\right.$ indiv. $\left./ \mathrm{m}^{2}\right)$, el Archipiélago San Lorenzo, B. C. $\left(0.033 \pm 0.004\right.$ indiv. $\left./ \mathrm{m}^{2}\right)$, e Isla San José, B. C. S. (0.029 \pm 0.003 indiv./ $\mathrm{m}^{2}$ ). Por el contrario, en Faro de Bucerías, Michoacán $\left(0.002 \pm 0.0007\right.$ indiv. $\left./ \mathrm{m}^{2}\right)$, hubo la más baja densidad junto con Tenacatita, Jalisco $\left(0.002 \pm 0.001 \mathrm{indiv} . / \mathrm{m}^{2}\right)$ e Isla Isabel, Nayarit $\left(0.003 \pm 0.001\right.$ indiv. $\left./ \mathrm{m}^{2}\right)$, y la especie estuvo ausente en dos sitios (San Jorge-Son. y las Islas Revillagigedo-Colima). El análisis estadístico mostró diferencias significativas entre los sitios K-W ${ }_{(20,1610)}=359.249, \mathrm{p}<0.001$ (Fig. 3) donde Bahía de los Ángeles, Loreto (B. C. S.), Archipiélago San Lorenzo, San Pedro Mártir (Sonora), Isla San José e Islas Marías (Nayarit) formaron un grupo común y diferente del resto de localidades al tener mayor densidad del pez.

El Ángel de Cortés, P. zonipectus fue más común en el norte, y estuvo presente en todos los sitios dentro del Golfo de California, pero solo se observó en tres localidades del Pacífico tropical mexicano (Islas Marías, el Faro de Bucerías y en las Islas Marietas, Jal.). Su promedio general de densidad fue de $0.006 \pm$ 0.0004 indiv. $/ \mathrm{m}^{2}$, y los sitios donde ésta fue más alta fueron las Islas Encantadas $(0.023 \pm 0.007$ indiv. $\left./ \mathrm{m}^{2}\right)$, Bahía de los Ángeles $(0.017 \pm 0.001$ indiv. $\left./ \mathrm{m}^{2}\right)$ y Archipiélago San Lorenzo (0.015 \pm 0.001 indiv. $\left./ \mathrm{m}^{2}\right)$, todos en Baja California. El análisis estadístico señaló diferencias significativas de las densidades entre los sitios (K-W $(20,1610)=399.311, \mathrm{p}<0.001$, Fig. 3), y para este caso Bahía de los Ángeles y Archipiélago San Lorenzo resultaron los sitios diferentes.

En relación con las especies cuyos datos no fueron graficados debido a sus bajas densidades a escala regional, como el Bocón puntos azules $O$. rosenblatti, presentó una distribución muy peculiar ya que solo fue visto en dos sitios arrecifales en el norte del golfo (Bahía de los Ángeles; $0.0009 \pm 0.0005$ indiv. $/ \mathrm{m}^{2}$ y Archipiélago San Lorenzo; $0.0007 \pm 0.0007$ indiv. $\left./ \mathrm{m}^{2}\right)$. Los valores de densidad de la especie (promedio regional de 0.0002 indiv. $/ \mathrm{m}^{2}$ ) fueron tan bajos que según el análisis estadístico, no hubo diferencias significativas en las poblaciones de éste pez a lo largo del occidente de México $\left(\mathrm{K}-\mathrm{W}_{(20,1610)}=18.679, \mathrm{p}=0.543\right)$. De manera similar, el Angel Clarión, Holacanthus clarionensis sólo se encontró en las Islas Revillagigedo $\left(0.027 \pm 0.003\right.$ indiv. $/ \mathrm{m}^{2}$ en dicha localidad $)$, y por ende la prueba de Kruskal-Wallis mostró diferencias significativas entre esa región y el resto de los sitios arrecifales de oeste de México $\left(\mathrm{K}-\mathrm{W}_{(20,1610)}=743.292, \mathrm{p}<0.001\right)$, dónde el Archipiélago Revillagigedo resultó diferente.

En cuanto a la riqueza por sitio de las especies de ornato pertenecientes a la NOM-059 (Fig. 3), en Isla San Jorge (Son.) solo apareció el Ángel Cortés ( $P$. zonipectus) con densidades bajas. Una riqueza tan baja (una especie) también fue observada en todas las localidades desde las Islas Marías (Nay.) hasta Huatulco (Oaxaca), con excepción de Manzanillo. En contraste, en Isla Tiburón (Son.), La Paz, Cabo Pulmo (B. C. S.) y Manzanillo (Col.) se registraron dos especies, mientras que en todo el centro y norte del Golfo de California (excepto San Jorge), los censos registraron al menos tres especies por sitio.

Relativo a la densidad por especie y sitio (Fig. 4), H. passer (Ángel rey) fue la especie dominante en 12 localidades de la región oeste de México (particularmente en zonas al sur de Cabo Pulmo, B. C. S.), mientras que en segundo lugar se observa la Damisela azul-amarillo (C. limbaughi) con altas densidades en cinco localidades del golfo, y una del Pacífico tropical (Manzanillo, Col.). De las restantes tres zonas, Isla San Jorge e Isla Tiburón (Son.) presentaron dominancia del Ángel de Cortés (P. zonipectus), y en las Islas Revillagigedo, el pez más común de los estudiados fue el Ángel Clarión, Holacanthus clarionensis.

Análisis económico de la captura: Los precios promedio de las especies de peces marinos en la NOM-059-SEMARNAT 2010 que se capturan en México, según las fuentes consultadas, fueron de 2.17 USD para la Damisela azul-amarillo (C. limbaughi), 9.07 USD del Ángel rey (H. passer), 13.58 USD del Ángel de Cortés (P. zonipectus), 13.84 USD 


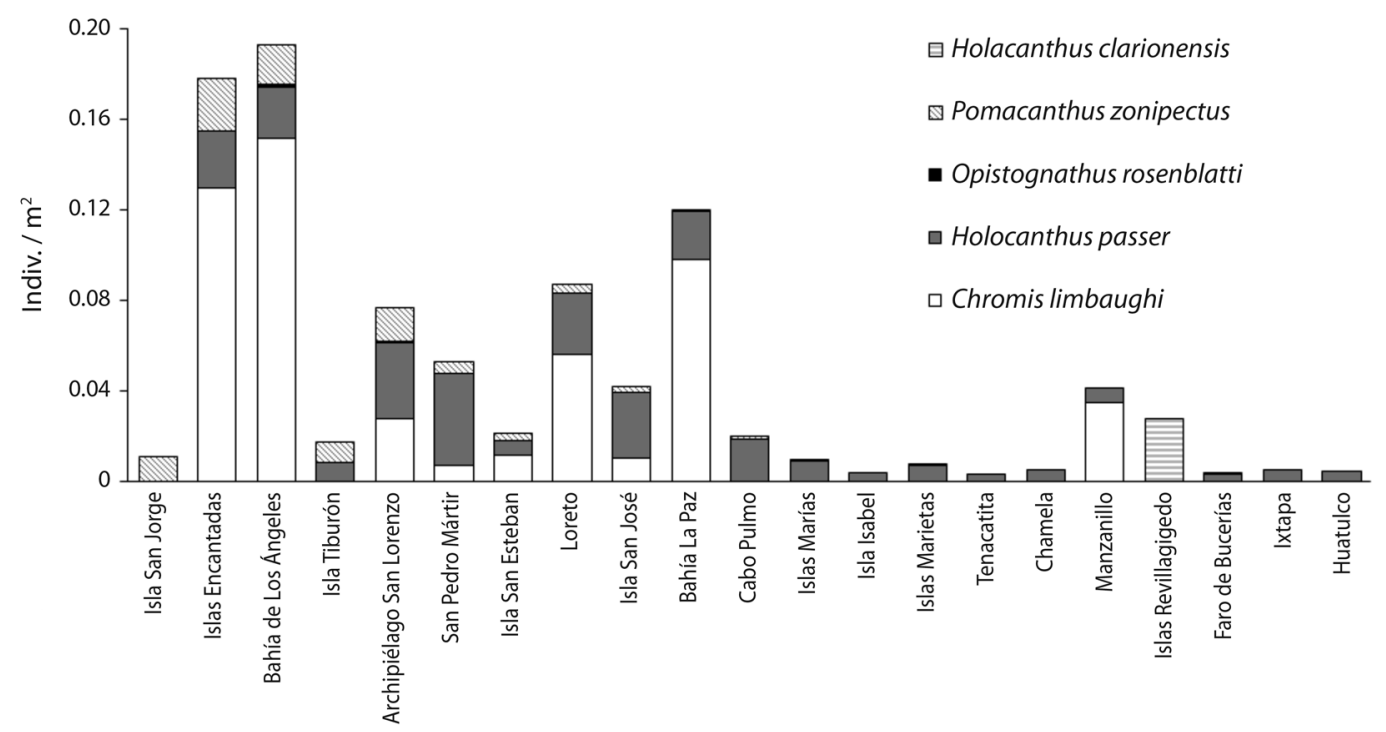

Fig. 4. Densidades poblacionales de las especies de interés, en cada localidad.

Fig. 4. Population densities of all the species of interest, at each locality.

para el Gobio puntos azules (O. rosenblatti), y 1000 USD del Ángel Clarión (H. clarionensis). Por otra parte, la captura total permisible para cada especie fue (en orden descendiente) de 0.0921 para $P$. zonipectus, 0.0622 de H. clarionensis, 0.0449 de H. passer, 0.0418 O. rosenblatti y 0.0312 para $C$. limbaughi. Al tomar estas cifras en cuenta, junto con las densidades poblacionales de cada especie por sitio, los resultados indicaron que la pesca de ornato de especies protegidas podría tener una ganancia bruta promedio (USD por temporada en un área de captura de 220 ha) de $207092 \pm 100441$ USD (Cuadro 1). Las localidades con un mayor potencial económico dentro del Golfo de California fueron Islas Encantadas, Bahía de los Ángeles, Archipiélago San Lorenzo (en Baja California) y San Pedro Mártir, en Sonora (Cuadro 1), con un rendimiento económico que variaba entre 51534 y 105963 USD (promedio de 81830 USD para las cuatro localidades). Fuera del golfo (donde actualmente no hay pesca de ornato) los arrecifes que pueden ofrecer mayor rentabilidad potencial fueron Manzanillo (10649 USD al año) y las Islas Marías (7912 USD anuales), pero las cifras superiores se calcularon para las Islas Revillagigedo (más de 3 millones USD por temporada anual de captura), debido a la dominancia del pez Ángel Clarión, que como se mencionó, que tiene un valor de mercado notablemente alto.

La ganancia neta promedio por temporada y en 220 ha que la pesca de ornato puede arrojar en el Pacífico Mexicano es de $43489 \pm$ 21092 USD en promedio (Fig. 5). En cuanto a las localidades en las que dicha pesca podría representar un mayor valor económico están las Islas Encantadas, Bahía de los Ángeles, San Lorenzo y San Pedro Mártir, todas en el norte del Golfo de California (Cuadro 1) con un rendimiento económico entre 10830 y 22282 USD. Fuera del golfo, las zonas con mayor potencial de rentabilidad son Manzanillo (derrama económica posible de 242 USD), y las islas Revillagigedo con más de 600 mil USD/temporada pesca/220 hectáreas.

\section{DISCUSIÓN}

La pesca de organismos ornamentales dentro del Golfo de California es una actividad económica viable y con potencial para el desarrollo económico de las comunidades ribereñas en las costas del Pacífico Mexicano 


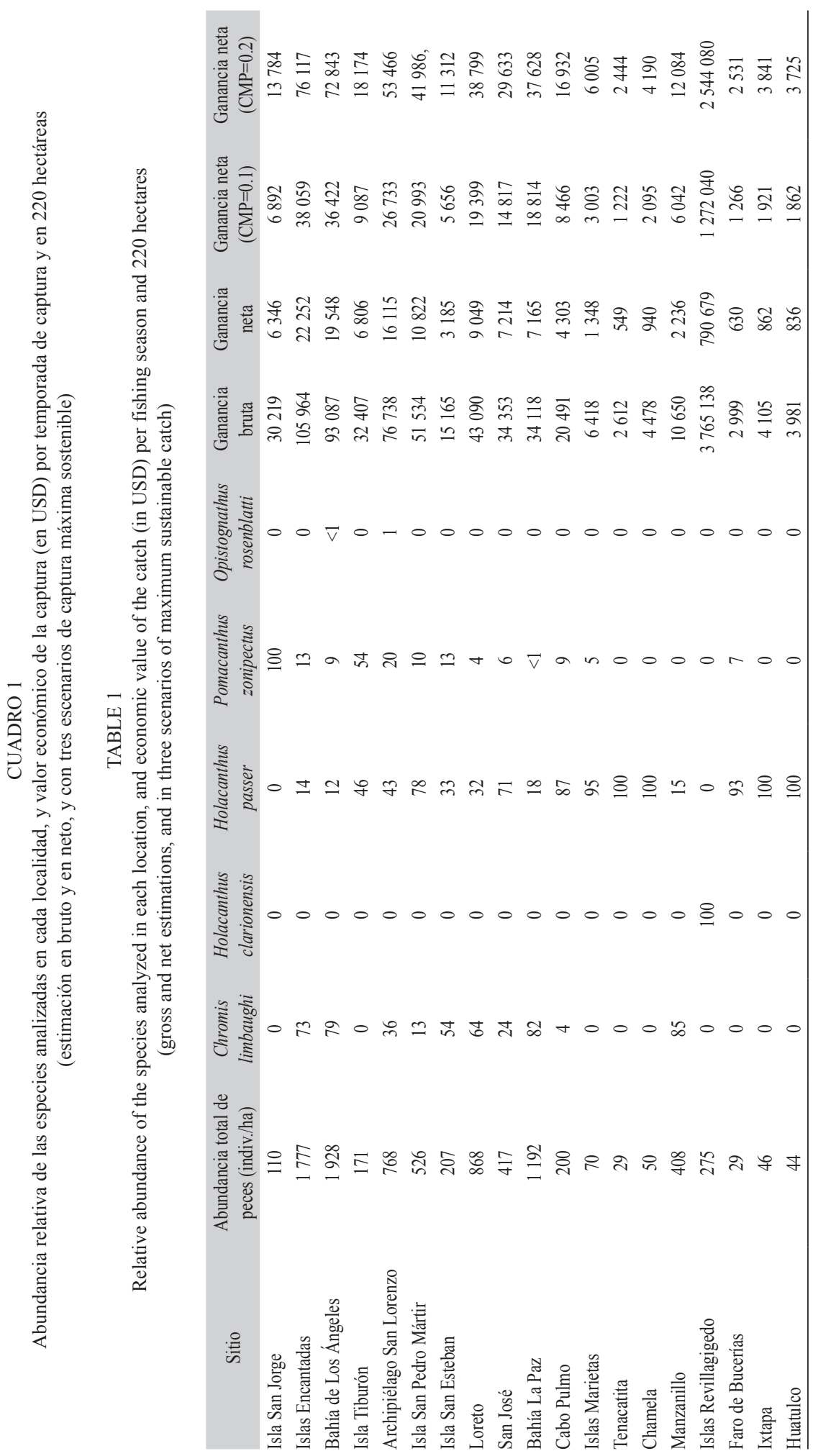




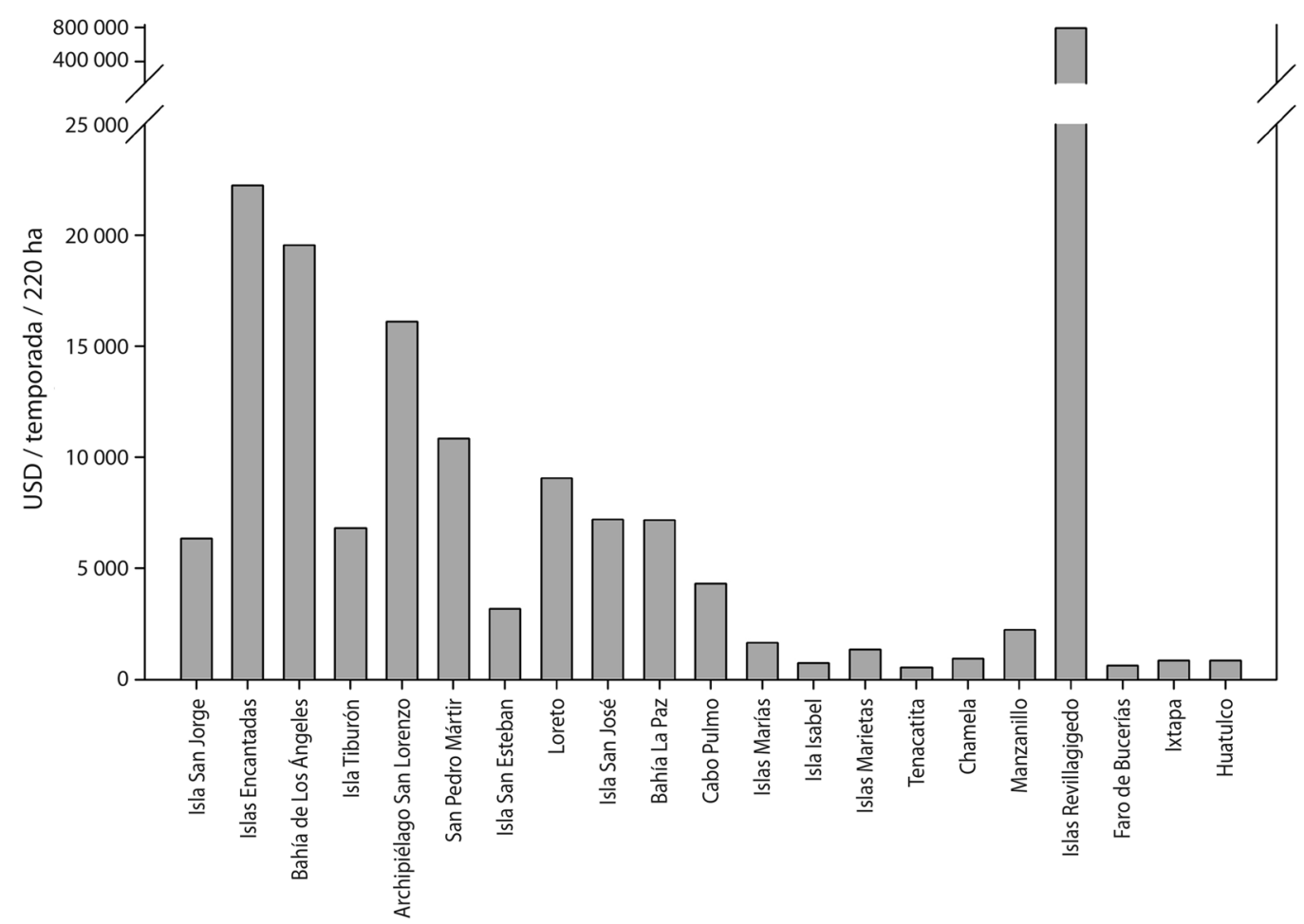

Fig. 5. Valor económico por localidad (en dólares por temporada de captura, y en 220 hectáreas de arrecife).

Fig. 5. Economic value per locality (in dollars per fishing season and 220 hectares of reef).

(Almenara-Roldán, 2000); además, gracias a las regulaciones actuales en este rubro, para zonas como Loreto o Bahía de Los Ángeles, la pesquería ornamental se encuentra en un nivel relativamente sustentable (Reyes-Bonilla et al., 2009). Sin embargo, debe tenerse en cuenta que el beneficio económico resultante para las organizaciones sociales (pescadores o cooperativas) que llevan a cabo la actividad es meramente aceptable. Es decir, como se demostró, la cantidad neta generada en una superficie de 220 hectáreas por la pesca de ornato de taxa incluidos en la NOM-059-SEMARNAT 2010 (los de mayor valor de mercado), no es tan alta como para que las cooperativas pudieran depender totalmente de ella, para cubrir sus necesidades financieras. Esta situación tiene tres motivos fundamentales: el área de captura relativamente pequeña que se otorga a las Unidades de Manejo, la vulnerabilidad intrínseca del recurso (razón por la cual el método de estimación de captura máxima aceptable arrojaba recomendaciones de pesca en porcentajes menores al $10 \%$ de la población residente para todos los casos), y finalmente, debido a que el mercado está controlado por el comprador; es decir, aunque el pez se extraiga, si no hay un cliente contactado de antemano, el organismo no puede ser comercializado (Germain et al., 2015). En tales condiciones, las ganancias reales están a expensas de las condiciones económicas en otros países, y como ejemplo, durante la crisis económica de los Estados Unidos a fines de la década pasada, la compra de peces de ornato disminuyó sensiblemente (C. Cázares. Buzo de la Sociedad Cooperativa "Mujeres del Golfo", com. pers.). Tomando en cuenta todo lo anterior, puede afirmarse que la pesca de ornato de especies protegidas en el occidente de México debe verse como una actividad económica complementaria para los grupos 
organizados de pescadores, más que como un medio de subsistencia en sí mismo.

A manera de ejemplo de la ganancia real que se obtuvo de la pesca ejercida sobre especies bajo la categoría de "Protección especial" según la NOM-059, una revisión de los permisos otorgados por SEMARNAT en el periodo de 2005-2009 (facilitados por dicha secretaría gracias a una solicitud al Instituto Federal de Acceso a la Información y Protección de Datos, IFAI) mostró que el aprovechamiento económico bruto promedio por sociedad cooperativa fue de 319817 USD. La especie mejor vendida fue el Ángel rey $H$. passer, seguido del Gobio puntos azules $O$. rosenblatti y el Ángel de Cortés P. zonipectus (197 347 USD); dicho orden de cantidad de capturas coincide con lo reportado por Reyes-Bonilla y colaboradores en 2009. No obstante ello, la especie que generó más divisas fue el Ángel Clarión (H. clarionensis), lo cual dio como resultado que de las cuatro sociedades cooperativas que aprovechan el recurso ornato dentro del Golfo de California ("Buzos del Golfo", "San Rafael y San Francisquito", "Mujeres del Golfo" y "Mar de Cortes"), la primera fue la que obtuvo las mayores ganancias debido a que es la única con permiso para la captura del Ángel Clarión. La información presentada mostró que la estimación promedio lograda en la presente investigación (207 092 USD de ganancia bruta anual para todos los sitios), es inferior a la registrada por SEMARNAT, y ello se debe en parte a que varias de las cooperativas emplean superficies mayores a 220 hectáreas para realizar sus actividades, y además a que para calcular el promedio citado, se incluyeron muchas zonas donde la densidad poblacional de las especies objetivo es muy baja, como la Isla San Jorge y la Isla Tiburón, en Sonora.

Como indican los resultados, las aguas del Golfo de California mantienen una gran diversidad de especies marinas, y en ciertos sitios se presentan altas densidades de las especies demandadas en el mercado acuarístico internacional. Pero además de ello, en la porción central del Pacifico Mexicano también existen sitios con buen potencial para la pesquería de ornato, en específico Manzanillo, Colima, y las Islas Marietas, Jalisco. Ahí están presentes especies de interés comercial (en especial la Damisela azul-amarillo C. limbaughi, el Ángel rey H. passer y al Ángel Cortés P. zonipectus) que en conjunto podrían generar ganancias netas de 2336 y 1347 USD por 220 ha de zona de captura, respectivamente. Cabe señalar que según Chávez-Comparán et al. (2010) a mediados de la década pasada, la densidad de $H$. passer era muy superior a la encontrada en nuestros censos $\left(2.2\right.$ indiv. $/ \mathrm{m}^{2}$ en ese trabajo, contra 0.006 indiv. $/ \mathrm{m}^{2}$ ). No está clara la razón del descenso del tamaño poblacional de esta especie en Manzanillo, pero no obstante ello, el análisis realizado aquí indica que la ganancia potencial es adecuada, y la actividad podría realizarse $\sin$ menoscabo de la salud de la población local, si se respeta la tasa de captura aquí sugerida.

El gobio puntos azules (O. rosenblatti), una especie endémica del Golfo de California, es una de las más buscadas a nivel internacional por el gran atractivo de su coloración y los impresionantes ritos nupciales que presentan los adultos. Además, su tamaño pequeño (menos de 10 cm; Piña-Espallargas et al., 2000) y su capacidad para adaptarse a la vida en cautiverio, hacen a esta especie muy demandada por los acuaristas (Almenara-Roldan, 2000), por lo que dicho pez está siendo extraído por todas las cooperativas que tienen su sede en el Golfo de California. No obstante ello, la especie presentaba densidades demasiado bajas en los parches arrecifales de los sitios de muestreo. El hábitat típico de residencia de $O$. rosenblatti se restringe a las superficies arenosas en el borde arrecifal, las cuales fueron submuestreadas durante los censos, ya que el interés se centraba en las especies presentes cerca de los corales o las formaciones rocosas. Por este motivo, nuestras estimaciones de abundancia y por ende, de captura máxima sostenible, son menores a las reales; así, los valores arrojados por la presente investigación representan una cuota baja, que puede ser usada con fines preventivos para conservar las poblaciones. Independientemente de lo anterior, aquí hacemos la sugerencia 
que la autoridad mantenga o disminuya los niveles de captura asignados a la fecha para $O$. rosenblatti (o incluso haga cierres parciales de zonas o temporales), ya que además de que por sí sola la especie no es suficientemente rentable, su captura parece haber provocado un impacto negativo en las poblaciones.

Otra de las especies más demandadas y abundantes dentro del Golfo de California es el Ángel de Cortés, P. zonipectus, debido al gran colorido que exhibe en su fase juvenil (Almenara-Roldan, 2000). Esta especie se distribuye desde el Golfo de California hasta Perú, y las Islas Galápagos (Allen \& Robertson, 1994), sin embargo las densidades encontradas en el Pacífico Mexicano fueron muy bajas. Registros de bibliografía anotan que sus números poblacionales siempre fueron relativamente bajos (Pérez-España, Galván-Magaña, \& Abitia-Cárdenas, 1996), y por ello, aquí se sugiere que la extracción comercial de dicha especie debe mantenerse dentro de los niveles en los que actualmente se efectúa, y que si la pesca de ornato se abre en localidades fuera del golfo, esta sea en niveles mucho menores a los que actualmente se realiza en ese mar interior.

Un sitio de máxima relevancia potencial para la pesca de ornato es el Archipiélago de Revillagigedo. Esta Reserva de la Biosfera alberga a una especie endémica, el Ángel Clarión (H. clarionensis), la cual es muy buscada por su gran colorido, rareza, lo restringido de su distribución, alcanza valores muy altos en el mercado (Almenara-Roldán, 2000). Algunos autores indican que por el precio de este pez, y el hecho que su pesca está prohibida por ley excepto por tres a cuatro permisos otorgados en todo México (en los cuales solo pueden atrapar organismos dentro del Golfo de California; Reyes-Bonilla et al., 2009), la pesca de la especie podría ser practicada de manera ilegal desde hace más de una década en la reserva (Wood, 2001). Los argumentos esgrimidos son la disminución aparente de las poblaciones en las Islas Revillagigedo (Almenara-Roldán \& Ketchum, 1994), el hecho que aunque se tienen registros de presencia de esta especie en la región de Cabo San Lucas hasta La Paz, ellos son ocasionales (Almenara-Roldán, 2000), y finalmente porque en el Golfo de California no existen poblaciones residentes de la especie en las cuales basar la pesca (Reyes-Bonilla et al., 2009). Además, Chávez-Comparan y colaboradores (2010) realizaron un estudio en Isla Socorro en 2008 (la isla más grande de las Revillagigedo) y obtuvieron una densidad de 2.2 indiv. $/ \mathrm{m}^{2}$, lo cual es mucho mayor que la observada en este estudio $\left(0.027\right.$ indiv. $\left./ \mathrm{m}^{2}\right)$, que fue obtenida en 2010; esa evidencia también apunta a que el Ángel Clarión está bajando sus densidades poblacionales en el archipiélago, y una de las razones de ello puede ser la pesca ilegal dentro de la reserva. Por todo lo anterior, sería recomendable buscar alternativas a la captura en campo, posiblemente propiciando su cultivo en laboratorio.

Proponer una solución a este problema es complejo, ya que aunque parece haber existido efecto de la pesca ilegal sobre las poblaciones del Ángel Clarión, nuestros resultados indican que aún con los valores de densidad poblacional de 2010, ésta pesquería resultaría rentable si se condujera ordenadamente dentro de las Revillagigedo, y que la derrama económica que la actividad puede arrojar es superior a los tres millones de dólares. Una situación semejante ocurrió en el caso del pepino café de mar (Isostichopus fuscus (Ludwig, 1875)), otra especie incluida en la NOM-059, y cuya pesca estuvo cerrada por varios años luego de que ocurriera una disminución de sus poblaciones a principios de los 1990s (Herrero-Pérezrul \& Chávez, 2005). La temporada de prohibición total dio lugar a un alto nivel de pesca ilegal, sin embargo el estado de las poblaciones era desconocido ya que no existían estudios poblacionales. Para resolver éste conflicto, la opción que dio la DGVS fue la de re-abrir la pesca dentro de un Predio Federal de Aprovechamiento otorgado a cooperativas, cuyo nivel de captura se definía anualmente dentro de una Mesa Técnica donde participaban permisionarios, autoridades federales y estatales, y académicos. El resultado fue que las poblaciones, aunque menores en número a lo que estuvieron en los 1980s, ya se estabilizaron y la actividad genera buenos 
dividendos para las comunidades ribereñas en Baja California y Baja California Sur (Shepherd, Martinez, Total-Granda, \& Edgar, 2004; Reyes Bonilla et al., 2009). Considerando este antecedente, los autores sugerimos que una estrategia similar (es decir, la apertura de la pesquería del Ángel Clarión en las Islas Revillagigedo, pero conducida de manera muy controlada, se evitaría exceder el porcentaje total de captura permisible, y manteniendo los estándares de calidad para la captura y traslado de los organismos) resultaría viable y traería notables beneficios económicos a las asociaciones productivas que la llevaran a cabo.

Por otra parte, puede verse que aunque el número de especies de peces incluidos en la NOM-059 fue muy similar por localidad tanto dentro como fuera del Golfo de California, la relevancia económica de la actividad tiene mucho mayor potencial en esta última región, debido a que ahí se tuvieron las mayores densidades; esta es la explicación por la cual los pescadores y permisionarios residentes de localidades dentro del golfo han pugnado por obtener permisos de pesca de fomento para dicha actividad.

Finalmente, la apertura de una pesquería de ornato en el Pacífico tropical mexicano podría enfrentar problemas independientes de la abundancia del recurso, de las idiosincrasias del mercado y de la pesca ilegal que potencialmente puede surgir. Entre ellos podría hablarse que por la falta de preparación y experiencia de los pescadores y los técnicos de un Predio Federal de Aprovechamiento, puede haber sobrepesca y mal manejo de los organismos, circunstancias que traerían pérdidas innecesarias a los productores y daños a las poblaciones naturales. Para evitar esto, las comunidades ribereñas interesadas, deberían de capacitarse con agencias de gobierno u organizaciones civiles de manera previa a iniciar sus actividades, con el fin de que se les dé a conocer las técnicas que se llevan a cabo en este tipo de pesquería, y paralelamente, generar conciencia para el cuidado y sustentabilidad del medio. No obstante ello, en zonas de muy baja abundancia del recurso, como Isla Isabel (Nay.), Tenacatita
(Jal.) y Faro de Bucerías (Mich.), no se recomendaría practicar este tipo de pesca, ya que no proporcionaría verdaderos beneficios a los pobladores de esas comunidades y causaría posibles daños a las comunidades arrecifales.

Es importante señalar que algunos autores han expresado preocupación sobre los efectos de la extracción de especies de ornato en la estructura y el funcionamiento de los sistemas arrecifales (Sala y Sánchez-Ortiz. 2000; Wood, 2001). Aunque la inquietud general es válida, en el caso del Pacífico Mexicano la situación no es tan grave ya que las especies de mayor interés pesquero no son fundamentales desde la perspectiva ecológica al ser relativamente poco abundantes, y no ser carnívoros superiores (Thomson et al., 2000), además se debe tomar en cuenta que los permisos de colecta son otorgados para la extracción de cantidades pequeñas de organismos (normalmente menos de 500 en un área de más de 200 hectáreas). Como indicaron Reyes-Bonilla et al. (2009) y Germain et al. (2015), la aplicación de reglas claras y el control de la cantidad de organismos capturados, puede asegurar la sostenibilidad de la pesquería de ornato en la región del Golfo de California, por lo que el seguir estos lineamientos ayudaría a hacer lo propio en el caso de nuevas pesquerías que se pudieran establecer en otras zonas del país., las cuales, al ser complementarias a otras actividades, podrían ser de gran utilidad para impulsar el desarrollo económico de los residentes de comunidades costeras.

Para concluir, según nuestro análisis, el desarrollo de la pesquería de especies de peces marinos de ornato pertenecientes a la NOM059-SEMARNAT 2010, que ya se practica en el Golfo de California, podría generar cantidades netas en el orden de los miles de dólares para las localidades ribereñas en otras partes del país donde se ejecute. Las localidades con un mayor potencial económico dentro del golfo fueron las Islas Encantadas, B. C., Bahía de los Ángeles, B. C. y el Archipiélago San Lorenzo, B. C., y en el Pacífico tropical, las zonas de Manzanillo, Col., las Islas Marietas, Jal., y sobre todo las Islas Revillagigedo, 
donde podría desarrollarse la pesquería del Ángel Clarión, especie con un valor extremadamente alto en el mercado. A pesar de lo anterior, los montos económicos potenciales no son suficientes como para sostener las necesidades financieras del grupo organizado que la practique; más bien, la pesca de ornato debe verse como una actividad alternativa. Finalmente, se recomienda que para que la pesquería de ornato sea una actividad rentable y sostenible, debe hacerse uso de las regulaciones ya puestas en operación, que ayudarán a controlar los niveles de cuotas de captura por área y especie; de esta forma se puede garantizar la sostenibilidad ecológica a largo plazo de esta labor.

\section{AGRADECIMIENTOS}

Este trabajo se generó con apoyo parcial del proyecto CONABIO MM003 (a Héctor Reyes Bonilla). Durante la realización del manuscrito, uno de los coautores de la nota (Francisco J. Fernández-Rivera Melo) recibió una beca de maestría otorgada por el Consejo Nacional de Ciencia y Tecnología (registro 274238).

\section{RESUMEN}

La pesca de ornamento es una actividad económicamente viable en países en desarrollo debido a que el acuarismo se ha convertido en un pasatiempo mundial. En México la pesca se ha dado desde los 80 's, especialmente en la Península de Baja California, pero no se tiene registro adecuado de la producción comercial, ni de la ganancia económica que se obtiene de la explotación del recurso. El objetivo de este estudio fue calcular el nivel máximo de captura permisible de las cinco especies de peces de ornato incluidas en la NOM-059-SEMARNAT 2010 (protegidas por mandato federal), y la ganancia neta que el recurso podría generar. Se realizaron censos $(\mathrm{N}=1610)$ en 21 localidades arrecifales del Pacifico mexicano y el Golfo de California, para estimar las densidades poblacionales de cada taxón; también se calculó su tasa de mortalidad natural $(\mathrm{M})$ a partir de la talla máxima $(\mathrm{L} \infty)$, constante de crecimiento (k) y la temperatura $(\mathrm{C})$, y la captura máxima permisible a partir de M. Finalmente, con los precios que se pagan en playa a los pescadores se hizo una estimación del valor económico que posee cada localidad en una temporada de pesca dada, y dentro de una superficie de 220 hectáreas (representativa del tamaño promedio de la zona de captura asignada a una cooperativa en México).
Los resultados indicaron que las localidades en las que la pesca de ornato tiene un mejor potencial económico son las Islas Encantadas, Bahía de los Ángeles y el Archipiélago San Lorenzo (todas en el estado de Baja California). Fuera del golfo (donde no hay pesca legal de ornato actualmente), la mayor rentabilidad potencial la presentan Islas Marietas (Jalisco), y las Islas Revillagigedo. La pesca de ornato puede ser una actividad viable en varias de las zonas analizadas del país, sin embargo, los montos potenciales que la captura representa no son suficientes como para sostener un grupo organizado que la practique; por ello, la pesca de ornato debe verse como una actividad comercial alternativa. Finalmente, para que ésta sea una pesquería rentable y sostenible, se recomienda hacer uso de regulaciones federales ya en existentes, que ayuden a controlar cuotas de captura.

Palabras clave: Holacanthus clarionensis, Holacantus passer, Pomacanthus zonipectus, Chromis limbaughi, Opistognathus rosenblatti, MAQTRAC.

\section{REFERENCIAS}

Albaladejo, V. D., \& Corpuz, V. T. (1981). A market study of the aquarium fish industry of the Philippines: an assessment of the growth and the mechanics of the trade. En E. D. Gomez, C. E. Birkeland, R. W. Buddemeier, R. E. Johannes, J. A. Jr. Marsh, \& R. T. Tsuda (Eds.), The reef and man. Proeedings of the fourth International Coral Reef Symposium (pp. 75-81) Philippines, Manila: University of Philippines.

Allen, G. R., \& Robertson, D. R. (1994). Fishes of the tropical eastern Pacific. USA: University of Hawaii Press.

Almenara-Roldán, S., \& Ketchum, J. T. (1994). Forgotten islands of the Mexican Pacific. OFI Journal, 9, 12-14.

Almenara-Roldán, S. C. (2000). Demanda internacional de especies marinas ornamentales del Golfo de California. En C. A. Sánchez-Ortiz, \& O. Aburto-Oropeza (Eds.), Recursos Arrecifales del Golfo de California, estrategias de manejo para las especies marinas de ornato (pp. 39-47). Estados Unidos: UABCSAcuario Birch.

Chávez-Comparán, J. C., Patiño-Barragán, M., CalderónRiveroll, G., Lezama-Cervantes, B., Ibarra Casillas, M., \& Bautista-Laureano, S. (2010). Listado de peces generado por censos visuales submarinos en la Isla Socorro, México. Revista Cubana de Investigaciones Pesqueras, 27(1), 72-78.

Food and Agriculture Organization of the United Nations (FAO). (1999). Ornamental aquatic life: what's FAO got to do with it? News \& Highlights. Recuperado el 05 de julio de 2016 de: http://www.fao.org/ News/1999/990901-e.htm 
Froese, R., \& Pauly, D. (2014). FishBase. Version, World Wide Web electronic publication (11/2014). Disponible en: http://www. fishbase. org

Gayanilo, Jr. F. C., Sparre, P., \& Pauly, D. (2005). FAOICLARM stock assessment tools II (FiSAT II). Rome, Italy: WorldFish Center, Food and Agriculture Organization of the United Nations.

Germain, N., Hartmann, H. J., Fernandez Rivera-Melo, F. J., \& Reyes-Bonilla, H. (2015). Ornamental reef fish fisheries: New indicators of sustainability and human development at a coastal community level. Ocean \& Coastal Management, 104, 136-149.

Giovanni, N. A. S. A. (2013). Ocean color radiometry online visualization and analysis. Recuperado el 5 de julio de: http://disc.sci.gsfc.nasa.gov/giovanni

Han, Z., Lai, L. W., \& Fan, J. (2002). The ornamental fish retail market in Hong Kong: its evolution and evaluation. Aquaculture Economics \& Management, 6(3-4), 231-247.

Herrero-Pérezrul, M. D., \& Chávez, E. A. (2005). Optimum fishing strategies for Isostichopus fuscus (Echinodermata: Holothuroidea) in the Gulf of California, Mexico. Revista de Biología Tropical, 53(3), 357-366.

Hodgson, G., \& Ochavillo, D. (2006). MAQTRAC marine aquarium trade coral reef monitoring protocol field manual. Californa, USA: Reef Check Foundation.

Lango Reynoso, F., Castañeda-Chávez, M., Zamora-Castro, J. E., Hernández-Zárate, G., Ramírez-Barragán, M. A., \& Solís-Morán, E. (2012). La acuariofilia de especies ornamentales marinas: un mercado de retos y oportunidades. Latin American Journal of Aquatic Research, 40(1), 12-21.

Pauly, D. (1983). Some simple methods for the assessment of tropical fish stocks. Manila, Philippines: FAO Corporate Document Repository.

Pérez-España, H., Galván-Magaña, F., \& Abitia-Cárdenas, L. A. (1996). Variaciones temporales y espaciales en la estructura de la comunidad de peces de arrecifes rocosos del suroeste del Golfo de California, México. Ciencias Marinas, 22(3), 273-294.

Piña-Espallargas, R. (2000). Especies marinas de ornato del Golfo de California y su uso. In O. Aburto-Oropeza \& C. . Sánchez-Ortiz (Eds.), Recursos arrecifales del Golfo de California. Estrategias de manejo para las especies marinas de ornato. Universidad Autónoma de Baja California Sur y Brich Aquarium at SCRIPPS (pp. 61-64). La Paz, México: UABCS.

Piña-Espallargas, R., Reyes-Bonilla, H., Ortuño-Manzanares, G., García-Núñez, N. E., Mendoza Vargas, L., \& González-Ania, L. V. (2000). Especies marinas de ornato del Golfo de California. En M. A. CisnerosMata, L. F. Beléndez-Moreno, E. Zárate-Becerra, M. T. Gaspar-Dillanes, L. C. López-González, C.
Saucedo-Ruíz (Eds.), Sustentabilidad y pesca responsable en México evaluación y manejo (pp. 879-914). México: INAPESCA-SAGARPA.

Piña Espallargas, R. (2005). La pesquería de especies marinas con fines de ornato en México. El Parque Marino de Loreto, BCS, como estudio de caso (Tesis de Maestría). Centro Interdisciplinario de Ciencias del Mar, Baja California, México.

Ramirez-Martínez, C., Mendoza-Alfaro, R., Aguilera González, C. J. (2010). Estado actual y perspectivas de la producción y comercialización de peces de ornato en México. Monterrey, México: INAPESCA/UANL.

Reyes-Bonilla, H., Herrero-Pérezrul, M. D., \& Fernandez Rivera-Melo, F. J. (2009). Aspectos económicos de los recursos pesqueros no tradicionales residentes en zonas arrecifales del Golfo de California. In J. Urciaga Garcia, L. F. Beltran Morales, \& D. Lluch Belda (Eds.), Recursos marinos y servicios ambientales en el desarrollo regional (pp. 245-264). La Paz, México: CIBNOR-UABCS-CICIMAR.

Sala, E., \& Sánchez-Ortíz, C. (2000). El "proyecto fauna arrecifal" y la investigación científica aplicada a la gestión de las especies marinas de ornato en el mar de Cortés. In O. Aburto-Oropeza \& C. Sánchez-Ortiz (Eds.), Recursos arrecifales del Golfo de California. Estrategias de manejo para las especies marinas de ornato. Universidad Autónoma de Baja California Sur y Brich Aquarium at SCRIPPS. la edición (pp. 139). La Paz, México: UABCS.

Secretaría de Medio Ambiente y Recursos Naturales (2012). Plan de Manejo Tipo para Peces Marinos de Ornato. México D.F.: SEMARNAT.

Shepherd, S. A., Martinez, P., Toral-Granda, M. V., \& Edgar, G. J. (2004). The Galápagos sea cucumber fishery: management improves as stocks decline. Environmental Conservation, 31(2), 102-110.

Sparre, P., \& Venema, S. C. (1997). Introducción a la evaluación de recursos pesqueros tropicales. Parte 1. FAO Documento Técnico de Pesca 306.1 Rev. 2. Santiago de Chile: IMPRESOS UNIVERSITARIA.

Thomson, D. A., Findley, L. T., \& Kerstitch, A. N. (2000). Reef fishes of the Sea of Cortes. The rocky-shore fishes of the Gulf of California. Texas, USA: University Texas Press, Arizona.

Wood, E. (2001). Collection of coral reef fish for aquaria: global trade, conservation issues and management strategies. Hereford,UK: Marine conservation Society.

Zar, J. H. (1999). Biostatistical Analysis (Vol. 53). New Jersey, USA: Prentice Hall. 